\title{
Giant ratchet magneto-photocurrent in graphene lateral superlattices
}

\author{
S. Hubmann $\odot,{ }^{1}$ V. V. Bel'kov, ${ }^{2}$ L. E. Golub $\odot,{ }^{2}$ V. Yu. Kachorovskii $\odot,{ }^{2,3}$ M. Drienovsky, ${ }^{1}$ \\ J. Eroms $\odot,{ }^{1}$ D. Weiss, ${ }^{1}$ and S. D. Ganichev ${ }^{1}$ \\ ${ }^{1}$ Terahertz Center, University of Regensburg, 93040 Regensburg, Germany \\ ${ }^{2}$ Ioffe Institute, 194021 St. Petersburg, Russia \\ ${ }^{3}$ CENTERA Laboratories, Institute of High Pressure Physics, Polish Academy of Sciences PL-01-142 Warsaw, Poland
}

(Received 9 April 2020; revised 4 June 2020; accepted 8 June 2020; published 3 August 2020)

\begin{abstract}
We report on the observation of the magnetic quantum ratchet effect in graphene with a lateral dual-grating top gate (DGG) superlattice. We show that the THz ratchet current exhibits sign-alternating magneto-oscillations due to the Shubnikov-de Haas effect. The amplitude of these oscillations is greatly enhanced as compared to the ratchet effect at zero magnetic field. The direction of the current is determined by the lateral asymmetry which can be controlled by variation of gate potentials in DGG. We also study the dependence of the ratchet current on the orientation of the terahertz electric field (for linear polarization) and on the radiation helicity (for circular polarization). Notably, in the latter case, switching from right- to left-circularly polarized radiation results in an inversion of the photocurrent direction. We demonstrate that most of our observations can be well fitted by the drift-diffusion approximation based on the Boltzmann kinetic equation with the Landau quantization fully encoded in the oscillations of the density of states.
\end{abstract}

DOI: 10.1103/PhysRevResearch.2.033186

\section{INTRODUCTION}

The discovery of graphene opened a new research direction in condensed matter physics. The unique optical properties of this material prompted a rapid development of photonics and optoelectronics. These are especially important for applications in the terahertz $(\mathrm{THz})$ range of frequencies; see e.g., Refs. [1-8]. THz-radiation-induced nonlinear optical effects, including rectification of $\mathrm{THz} /$ infrared electromagnetic waves, offer a new playground for many intriguingphenomena in graphene; see, e.g., reviews $[9,10]$. These phenomena deliver graphene-specific mechanisms of photocurrent generation and provide a basis for the development of novel graphene radiation plasmonic detectors. Such detectors are compact, tunable by gate voltage and have already shown fast and sensitive operation in a broad frequency band from sub- $\mathrm{THz}$ to infrared, and from ambient- to cryogenic temperatures.

Among the highly promising radiation detecting mechanisms is the ratchet effect, i.e., the generation of a dc electric current responding to an $a c$ electric field in systems with broken $P$ symmetry. This is one of the most general and fundamental nonlinear phenomena in optoelectronics; for reviews see, e.g., Refs. [11-14]. In graphene, the ratchet effect can be obtained in monolayers with asymmetric micropatterns [15-17], layers with built-in structure inversion asymmetry [18] (in this case it is typically called photogalvanic effect

Published by the American Physical Society under the terms of the Creative Commons Attribution 4.0 International license. Further distribution of this work must maintain attribution to the author(s) and the published article's title, journal citation, and DOI.
[11]), short-channel devices, like field effect transistors with asymmetric boundary conditions [1,19-25], as well as in structures with asymmetric grating type of electrodes [26-31]. Besides their fundamental significance, the two latter types of ratchets are extremely important for applications, since they provide a very promising route towards fast, sensitive, and gate-tunable detection of $\mathrm{THz}$ radiation at room temperature. The study of ratchet effects in graphene under different transport regimes such as the drift-diffusion $[28,29]$ or the hydrodynamic one [30-32], including plasmonic effects, is a very challenging task that has just begun to be explored.

For ratchets new physics comes into play, when an external magnetic field is applied. It has been shown that a ratchet effect can be induced by an external magnetic field even in case of homogeneous graphene with structure inversion asymmetry [33]. The effect was called magnetic quantum ratchet effect and belongs to the class of magneto-photogalvanic effects [34-37]. It is sensitive to disorder and tunable by a gate voltage. The observation triggered numerous theoretical proposals aimed to enhance and control magnetic ratchet effects in graphene-based systems. In particular, it was predicted that the magnetic ratchet effect can be enormously increased under cyclotron resonance condition and in periodic grating gate structures.

Here we report the observation of the giant oscillating magnetic ratchet effect in graphene with superimposed lateral superlattice, consisting of a dual-grating top gate (DGG) structure. We show particularly, that, the THz-radiation-induced ratchet current exhibits sign-alternating magneto-oscillations stemming from Landau quantization and having the same period as the Shubnikov-de Haas ( $\mathrm{SdH})$ oscillations. The amplitude of the ratchet current oscillations is greatly enhanced (at least by one order of magnitude) as compared to the ratchet 
effect at zero magnetic field previously studied in similar structures [29]. The latter effect was shown to be caused by the combined action of a spatially periodic in-plane potential and the spatially modulated light due to the near-field effects of the radiation diffraction [29]. Quantum oscillations appear also as a function of top or back gate voltage in our DGG structures when subjected to a constant magnetic field. Thereby, the direction of the current is controlled by the lateral asymmetry parameter $[11,29]$

$$
\Xi=\overline{|\boldsymbol{E}(x)|^{2} \frac{d V(x)}{d x}}
$$

Here the overline stands for the average over the ratchet period, $d V(x) / d x$ is the derivative of the coordinate-dependent electrostatic potential $V(x)$, and $\boldsymbol{E}(x)$ the distribution of the radiation electric field being coordinate dependent due to the near field of diffraction. We show that by changing the individual gate voltages of the dual-grating gate structure we can controllably change the sign of $\Xi$ and, thus, the direction of the ratchet current. Furthermore, we study the response to both linear and circularly polarized radiation and demonstrate that the magnetic ratchet current is sensitive to the orientation of the linear polarization with respect to the fingers of the DGG structure as well as to the radiation helicity in the case of circular polarization. In the latter case switching from rightto left-circular polarization results in a phase shift of the oscillations by $\pi$, i.e., at constant magnetic field, the current direction reverses. The theoretical modeling of our results is based on the experimental values of all involved parameters and the assumption that the frequency of the incoming radiation is much higher than the plasmonic frequency, so that plasmonic effects do not contribute substantially. We demonstrate that in strong quantizing magnetic fields all observations can be well fitted by the drift-diffusion approximation of the kinetic Boltzmann equation. Within this approach we find that the photocurrent is proportional to second derivative of the longitudinal resistance and, therefore, almost follows the $\mathrm{SdH}$ resistance oscillations with a large enhancement factor arising due to differentiating of rapidly oscillating function.

The paper is organized as follows. In Sec. II we describe the investigated samples and experimental technique. In Sec. III we discuss the observed magnetic ratchet effects generated by linearly and circularly polarized $\mathrm{THz}$ radiation. In the following Secs. IV and V we present the theory and compare the corresponding results with the experimental data. Finally, in Sec. VI we summarize the results.

\section{SAMPLES AND METHODS}

Single-layer graphene samples encapsulated in hexagonal boron nitride $(\mathrm{hBN})$ were prepared using the van der Waals stacking technique with $\mathrm{Cr} / \mathrm{Au}$ edge contacts established by Wang et al. [38]. After exfoliation and stacking on top of a silicon wafer with $285 \mathrm{~nm}$ thermal oxide, a Hall bar mesa was etched using $\mathrm{CHF}_{3}$ based reactive ion etching, and edge contacts were deposited by thermal evaporation. To avoid gate leakage at the mesa sidewalls, the samples were covered with $5 \mathrm{~nm} \mathrm{Al}_{2} \mathrm{O}_{3}$ using atomic layer deposition. The highly doped $\mathrm{Si}$ wafer serves as a uniform back gate.
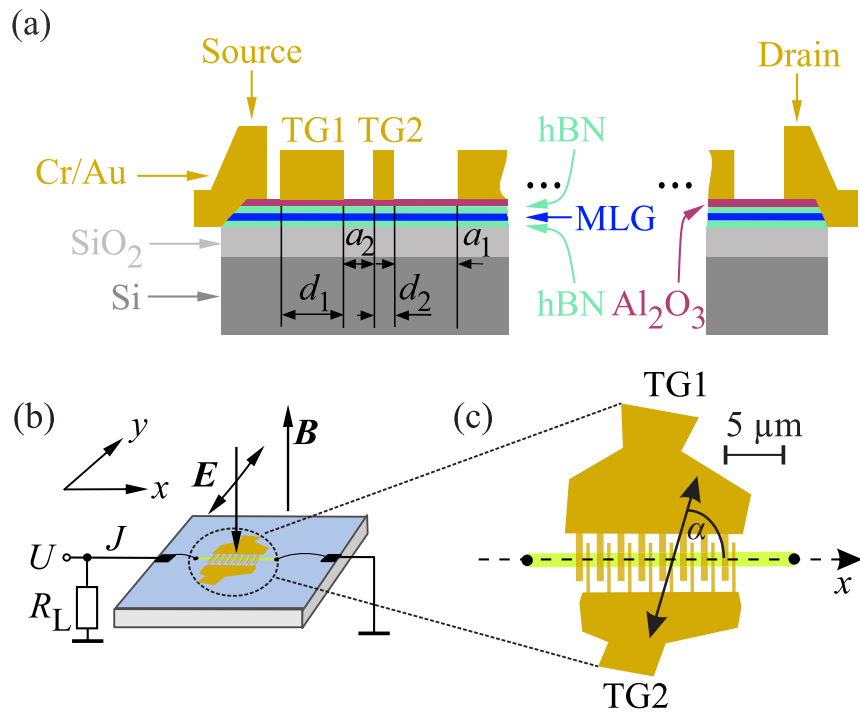

FIG. 1. (a) Cross section sketch of the sample structure and the dual-grating gate superlattice. (b) Sketch of the experimental setup for the ratchet photocurrent measurements. (c) Sketch of the superlattice (top view). Black dots show electric contacts to graphene layer.

Afterwards, following the recipe of Ref. [29], a dualgrating top gate (DGG) superlattice was fabricated on top of the $\mathrm{hBN} /$ graphene/hBN flakes for the measurement of the ratchet photocurrent. The micropatterned periodic DGG fingers were made by electron beam lithography and subsequent deposition of metal ( $5 \mathrm{~nm} \mathrm{Cr}$ and $20 \mathrm{~nm} \mathrm{Au}$ ) on graphene covered by $\mathrm{hBN}$ and a $5 \mathrm{~nm} \mathrm{Al}_{2} \mathrm{O}_{3}$ layer. A sketch of this superstructure is shown in Figs. 1(a) and 1(c). Two gate stripes with different widths $d_{1}=600 \mathrm{~nm}$ and $d_{2}=300 \mathrm{~nm}$ and spacings $a_{1}=600 \mathrm{~nm}$ and $a_{2}=300 \mathrm{~nm}$ in between form the supercell of the lattice. The cell is repeated eight times resulting in a superlattice with a total length of $14.4 \mu \mathrm{m}$. All wide stripes were connected forming multifinger top gate electrode TG1. Similarly connected narrow stripes formed gate electrode TG2; see yellow areas in Fig. 1(c). Independent bias voltages $\left(U_{\mathrm{TG} 1}, U_{\mathrm{TG} 2}\right)$ could be applied to wide and narrow gate stripes making the electrostatic potential asymmetry in the graphene tunable. The width of the whole structure is $1.4 \mu \mathrm{m}$ yielding the total area $A=14.4 \times 1.4 \mu \mathrm{m}^{2}=20.2 \mu \mathrm{m}^{2}$.

To measure the longitudinal resistance and the photocurrent normal to the DGG stripes Ohmic contacts were fabricated; see Fig. 1(c). Low-temperature transport measurements, which are possible in our structure in two-point configuration only, showed well-resolved $\mathrm{SdH}$ oscillations; see right inset in Fig. 2. The oscillatory part $\Delta R_{x x}(B)$ was obtained by subtracting a polynomial background of the form $A_{0}+C B^{2}+$ $D B^{4}$ from the longitudinal resistance $R_{x x}(B)$. The coefficients $A_{0}, C$, and $D$ were obtained by fitting to the high field data. Also a clear charge neutrality point was observed at $U_{\mathrm{BG}}=$ $-4 \mathrm{~V}$ while tuning the Fermi energy by sweeping the back gate (see left inset of Fig. 2), so that for $U_{\mathrm{BG}}>-4 \mathrm{~V}$ we have electron conductivity and for opposite inequality the hole one. At the charge neutrality point, a slight background doping of approximately $7 \times 10^{10} \mathrm{~cm}^{-2}$ is present due to the top gates. The variation of the back gate changes the charge carrier 


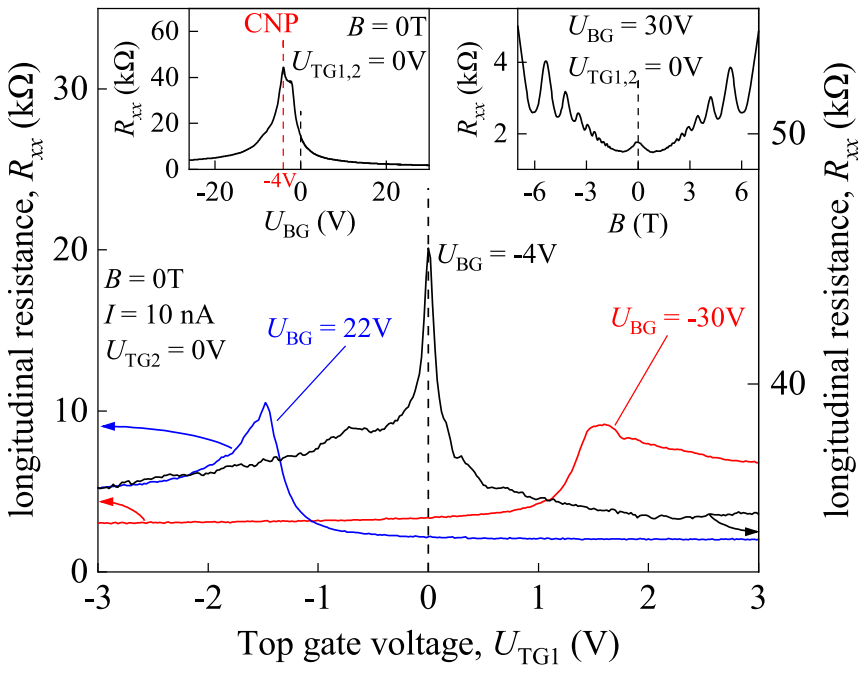

FIG. 2. Dependences of the longitudinal resistance $R_{x x}$ on the top gate voltage $U_{\mathrm{TG} 1}$ for different back gate voltages. Left and right insets show dependences of $R_{x x}$ on back gate voltage and magnetic field, respectively.

density in the range from a hole density of $1.5 \times 10^{12} \mathrm{~cm}^{-2}$ to an electron density of $1.9 \times 10^{12} \mathrm{~cm}^{-2}$ for the $U_{\mathrm{BG}}$ variation from -30 to $30 \mathrm{~V}$. We obtained an electron mobility $\mu=$ $29000 \mathrm{~cm}^{2} / \mathrm{Vs}$ and a hole mobility of $\mu=15000 \mathrm{~cm}^{2} / \mathrm{Vs}$ at $T=4.2 \mathrm{~K}$. Application of a positive top gate potential shifts the peak of the longitudinal resistance to higher negative $U_{\mathrm{BG}}$ while for high negative top gate voltages it moves to positive $U_{\mathrm{BG}}$; see Fig. 2 .

As a radiation source for our experiments a continuous wave methanol terahertz laser with a radiation frequency of $f=2.54 \mathrm{THz}(\hbar \omega=10.5 \mathrm{meV})$ and a radiation power $P$ of the order of $50 \mathrm{~mW}$ was used [39-41]. The radiation was focused onto the sample using an off-axis parabolic mirror resulting in a spot size of $\approx 1.3 \mathrm{~mm}$, which yields an intensity $I \approx 3.8 \mathrm{~W} \mathrm{~cm}^{-2}$. The radiation power coming onto the sample is calculated after $P_{\mathrm{S}}=I \cdot A$. The laser beam had an Gaussian shape as checked by a pyroelectric camera $[42,43]$. The radiation was modulated at about $75 \mathrm{~Hz}$ by an optical chopper in order to use standard lock-in technique.

The optically pumped molecular laser used here emits linearly polarized radiation. In our setup its polarization plane is oriented along the $x$ axis being normal to the dual-grating gate stripes. In experiments with linearly polarized radiation, the orientation of the radiation electric field vector $\boldsymbol{E}$ was varied by rotation of a mesh grid polarizer mounted behind the quarter-wave plate providing circularly polarized radiation. The azimuth angle $\alpha$ is the angle between the radiation electric field vector and the $x$ direction [Fig. 1(c)]. The Stokes parameters describe the degree of the linear polarization in the basis $(x, y)$ and the basis $(\tilde{x}, \tilde{y})$ rotated by $45^{\circ} P_{\mathrm{L}}$ and $\tilde{P}_{\mathrm{L}}$, respectively. In this setup, they are given by

$$
P_{\mathrm{L}}(\alpha)=\cos 2 \alpha, \quad \tilde{P}_{\mathrm{L}}(\alpha)=\sin 2 \alpha .
$$

In experiments with elliptically polarized radiation, the radiation helicity was varied by rotating the quarter-wave plate by the angle $\varphi$. By that, at $\varphi=0$ radiation is linearly polarized and $\boldsymbol{E}$ is parallel to $x$, whereas at $\varphi=45^{\circ}$ and $\varphi=135^{\circ}$ the radiation is circularly polarized with opposite helicities. In this setup the Stokes parameters are given by $[36,44]$

$$
\begin{aligned}
P_{\mathrm{L}}(\varphi) & =(\cos 4 \varphi+1) / 2, \quad \tilde{P}_{\mathrm{L}}(\varphi)=\sin 4 \varphi / 2, \\
P_{\text {circ }} & =\sin 2 \varphi,
\end{aligned}
$$

where $P_{\text {circ }}$ defines the degree of circular polarization. The ratchet photocurrents were measured in a magneto-optical cryostat at a temperature of $4.2 \mathrm{~K}$ as a voltage drop along a load resistor of $R_{\mathrm{L}}=100 \Omega$ using standard lock-in technique and then calculated using $J=U / R_{\mathrm{L}}$. In all graphs, the photocurrent is normalized to the radiation power coming onto the sample, $P_{\mathrm{S}}$. The values of normalized current $J / P_{s}$, which in our experiments lie in the range from about 1 to $10 \mathrm{~mA} / \mathrm{W}$, correspond to the measured current $J$ ranging from 1 to $10 \mathrm{nA}$. An external magnetic field with $B$ up to $7 \mathrm{~T}$ is applied normal to the graphene plane, as sketched in Fig. 1(b).

\section{RESULTS}

Before discussing our results on magnetic current we briefly address the photocurrents detected at zero magnetic field. In our previous work [29], in which we studied similar structures and applying terahertz radiation with the same parameters, we demonstrated that illumination of the DGG superlattice on graphene results in a photocurrent exhibiting characteristic behavior of the ratchet effect. In particular, photocurrent direction and magnitude: (i) are sensitive to the orientation of the radiation electric field vector $\boldsymbol{E}$ and/or the radiation helicity, (ii) depend on the carrier charge sign (electrons/holes), and (iii) are controlled by the lateral asymmetry parameter $\Xi$, which can be varied by applying voltages $U_{\mathrm{TG} 1}$ and $U_{\mathrm{TG} 2}$ to the individual subgates. Note that switching of the gate voltage from $U_{\mathrm{TG} 1}>0, U_{\mathrm{TG} 2}=0$ to $U_{\mathrm{TG} 1}=0$, $U_{\mathrm{TG} 2}>0$ leads to a change in the sign of $\Xi$ and, as a consequence, to a reversal of the photocurrent direction. Theoretical analysis carried out in Ref. [29] reveals that the photocurrent is caused by a combined action of a spatially periodic in-plane electrostatic potential and the radiation spatially modulated due to the near-field effects of the diffraction on the DGG stripes. Experiments and theory of this effect present a selfconsisted detailed picture of the ratchet current formation, therefore, in the present paper we focused on the magnetic ratchet effect in graphene.

Applying an external magnetic field we observed that the ratchet photocurrent drastically changes: The photocurrent exhibits sign-alternating $\mathrm{SdH}$-like magneto-oscillations with an amplitude by more than an order of magnitude larger than the photocurrent at zero magnetic field. A characteristic magnetic field dependence is shown in Fig. 3 for $U_{\mathrm{TG} 1,2}=$ 0 , radiation electric field oriented perpendicular to the gate stripes and back gate voltage $U_{\mathrm{BG}}=30 \mathrm{~V}$. Note that at zero top gate voltages, the lateral asymmetry is created by the built-in potential caused by the metal stripes deposited on top of graphene.

Comparing the magnetic-field dependencies of the ratchet photocurrent and the longitudinal resistance $R_{x x}(B)$ we found out that extrema positions of the photocurrent and the $\mathrm{SdH}$ oscillations coincide in weak fields. This is seen clearly in Fig. 3, where the left vertical dashed line exemplary indicates 


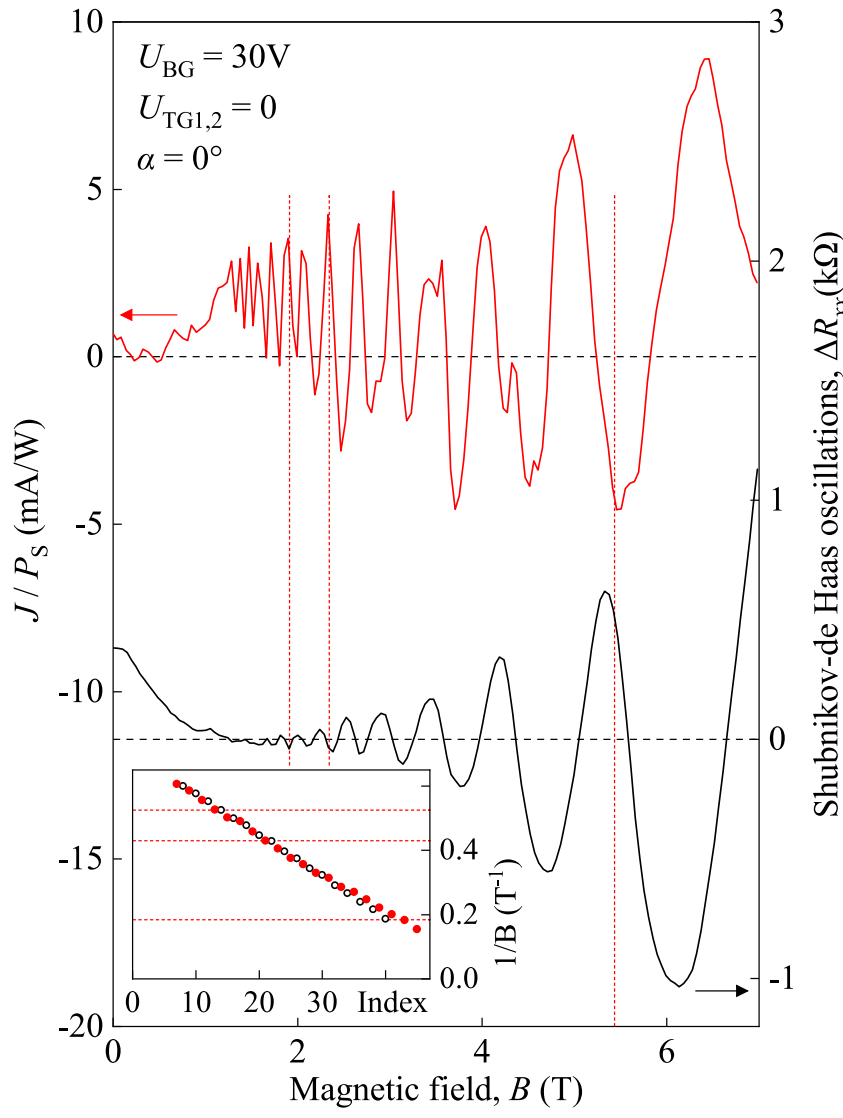

FIG. 3. The photocurrent normalized to the radiation power coming onto the sample, $P_{\mathrm{S}}$, and the $\mathrm{SdH}$ longitudinal resistance oscillations $\Delta R_{x x}$ as functions of magnetic field $B$. The oscillatory part $\Delta R_{x x}(B)$ was obtained by subtracting a polynomial background of the form $A_{0}+C B^{2}+D B^{4}$ from the longitudinal resistance $R_{x x}(B)$. The coefficients $A_{0}, C$, and $D$ were obtained by fitting to the high field data. Vertical dashed lines mark some of the oscillations' extrema positions. The inset shows corresponding positions of transport (black circles) and photocurrent oscillations (red dots) on a $1 / B$ scale as a function of the extremum's index. Horizontal dashed lines correspond to the vertical lines in the main plot.

extrema positions of the photocurrent and the $\mathrm{SdH}$ oscillations. It should be noted that, whereas at low magnetic fields the photocurrent oscillations follow $R_{x x}(B)$, at high magnetic fields a magnetic field-dependent phase shift is present; see the right vertical dashed line in Fig. 3. These fields are slightly higher than the magnetic field of the cyclotron resonance $B_{C R}=\omega \varepsilon_{\mathrm{F}} /\left(|e| v_{0}^{2}\right)$, where $\omega, \varepsilon_{\mathrm{F}}$, and $v_{0}$ are the radiation angular frequency $\omega=2 \pi f$, Fermi energy, and the Dirac velocity in graphene, respectively. Note that for the carrier density $n_{s} \approx 10^{12} \mathrm{~cm}^{-2}$, and radiation frequency $f=$ $2.54 \mathrm{THz}$, relevant to experiment, we estimated $B_{C R} \approx 1.8 \mathrm{~T}$.

The resistance oscillations can also be obtained at fixed magnetic field by the variation of the carrier density $n_{s}$, e.g., changing the back gate voltage $\left(n_{s} \propto U_{B G}\right)$. This kind of oscillations we also observed in the ratchet photocurrent. Figure 4 shows an example of such oscillations obtained for $B=5.8 \mathrm{~T}, U_{\mathrm{TG} 1,2}=0$, and for linear polarization with $\alpha=0$.

Now we turn to the results obtained by variation of the lateral asymmetry applying different voltages to the top subgates

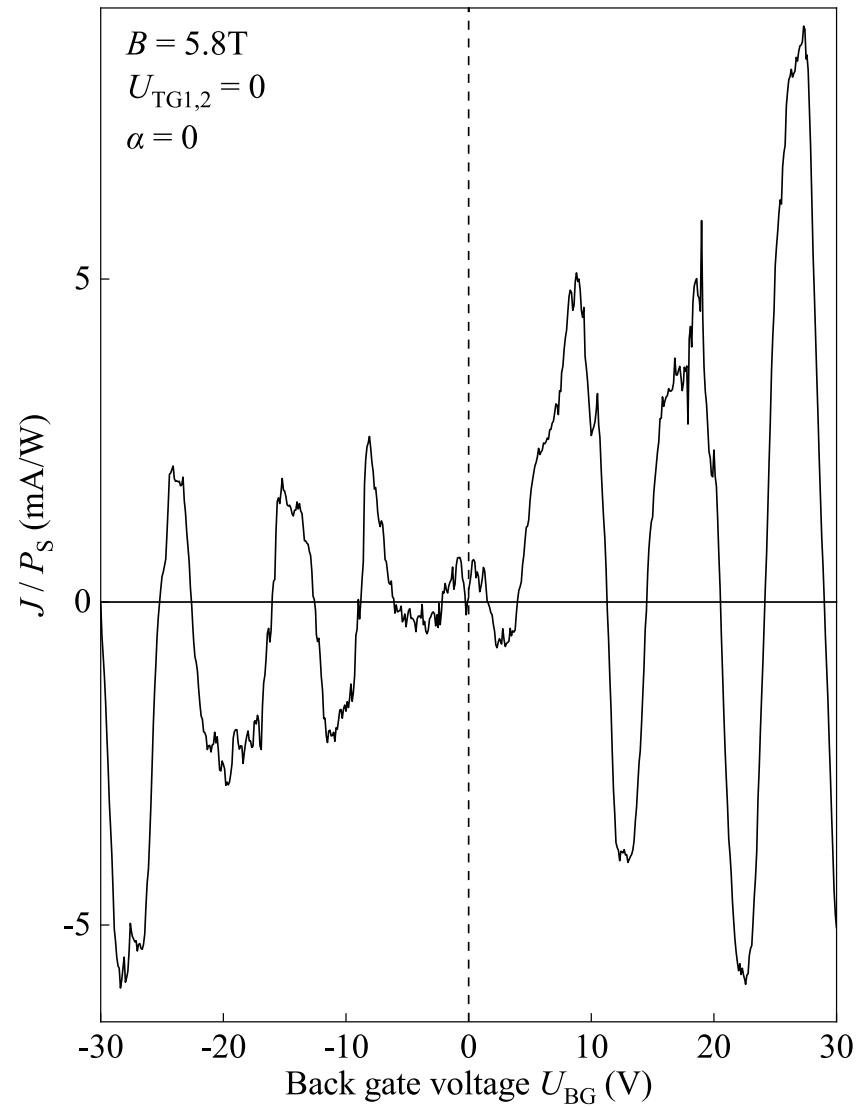

FIG. 4. Dependence of the ratchet photocurrent normalized to the radiation power coming onto the sample, $P_{\mathrm{S}}$, on the voltage applied to the back gate. The data are obtained for linear polarization with $\alpha=0$.

TG1 and TG2. Figure 5 shows magnetic ratchet photocurrent oscillations as a function of top gate voltage $U_{\mathrm{TG} 1}\left(U_{\mathrm{TG} 2}\right)$ obtained for zero biased top gate TG2 (TG1), and for linear polarization with $\alpha=0$. At zero top gate voltages the photocurrent has the same sign and almost the same amplitude. Sweeping voltage of one of the top gates while holding the other one at zero bias we observed that the photocurrent oscillates in a similar way as it is detected for the variation of back gate voltage. However, the period of oscillations is substantially decreased, which clearly follows from the different separation between top or back gates and graphene [45]. At high gate voltages corresponding to stronger lateral asymmetry as the built-in one we observed that maxima (minima) of the dependence on $U_{\mathrm{TG} 1}$ corresponds to minima (maxima) of the dependence on $U_{\mathrm{TG} 2}$. This is illustrated in Fig. 5 for positive and negative top gate voltages by vertical red/black dashed lines. It reveals that the change of sign of the lateral asymmetry parameter $\Xi$ results in the change of the oscillations sign, as expected for the ratchet effect. Indeed, e.g., for the top gate voltage combinations marked by the right vertical dashed lines $\left(U_{\mathrm{TG} 1}>0, U_{\mathrm{TG} 2}=0\right.$, red curve, and $U_{\mathrm{TG} 1}=0, U_{\mathrm{TG} 2}>0$, black curve) the signs of the asymmetry parameters $\Xi$ are opposite.

All results discussed previously were obtained for linearly polarized radiation with the electric field $\boldsymbol{E}$ perpendicular to the DGG stripes. Further experiments demonstrate that 


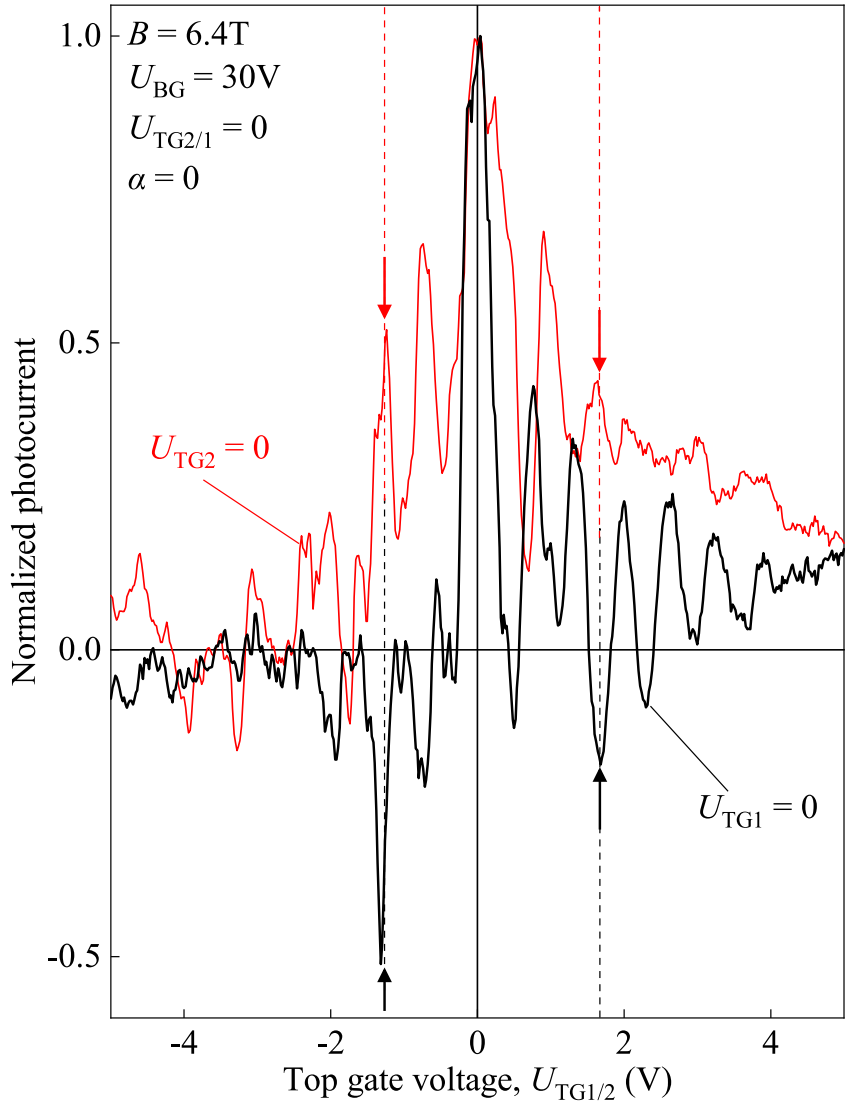

FIG. 5. Top gate voltage $U_{\mathrm{TG} 1}\left(U_{\mathrm{TG} 2}\right)$ dependencies of the photocurrent normalized to the photocurrent maximum. The curves were obtained varying $U_{\mathrm{TG} 1}\left(U_{\mathrm{TG} 2}\right)$ holding zero bias at the other subgate TG2 (TG1) and for linear polarization with $\alpha=0$. Since at high gate voltages maxima of one dependence correspond to minima at the other this provides an evidence for the ratchet effect being proportional to $\Xi$. This is illustrated by the vertical dashed lines at high positive (negative) gate voltages at which the lateral asymmetry introduced by the applied voltages is stronger than the built-in one caused by the metal stripes deposited on top of graphene.

magneto-oscillations of the ratchet current are sensitive to the orientation of the $\mathrm{THz}$ electric field vector; see Fig. 6. Our measurements demonstrate that dependence of the current on the direction of the linear polarization can be well fitted as

$$
J=J_{A} P_{\mathrm{L}}(\alpha)+J_{B} \tilde{P}_{\mathrm{L}}(\alpha)+J_{D},
$$

where $J_{A}, J_{B}$, and $J_{D}$ are magnetic field-dependent fitting parameters. Figure 6(b) exemplary shows the polarization dependence of the total photocurrent measured at fixed magnetic field $B=4.9 \mathrm{~T}$. The magnetic field dependence of the coefficients $J_{A}$ and $J_{D}$ yielding dominating contributions at low magnetic field are presented in Fig. 6(a). The curves in Fig. 6(a) were obtained from the magnetic field dependence of the photocurrent excited by the THz electric field vectors $\boldsymbol{E}$ oriented perpendicular $(\alpha=0)$ and parallel $\left(\alpha=90^{\circ}\right)$ to the stripes. For these angles the photocurrent contribution $J_{B} \tilde{P}_{\mathrm{L}}$ is zero and the total current is given by $J= \pm J_{A}+J_{D}$. Consequently, the magnetic field dependencies of $J_{A}$ and $J_{D}$ were calculated, respectively, as a half-difference and half-sum of the photocurrents measured for $\alpha=0$ and $90^{\circ}$. Figure 6 (a)
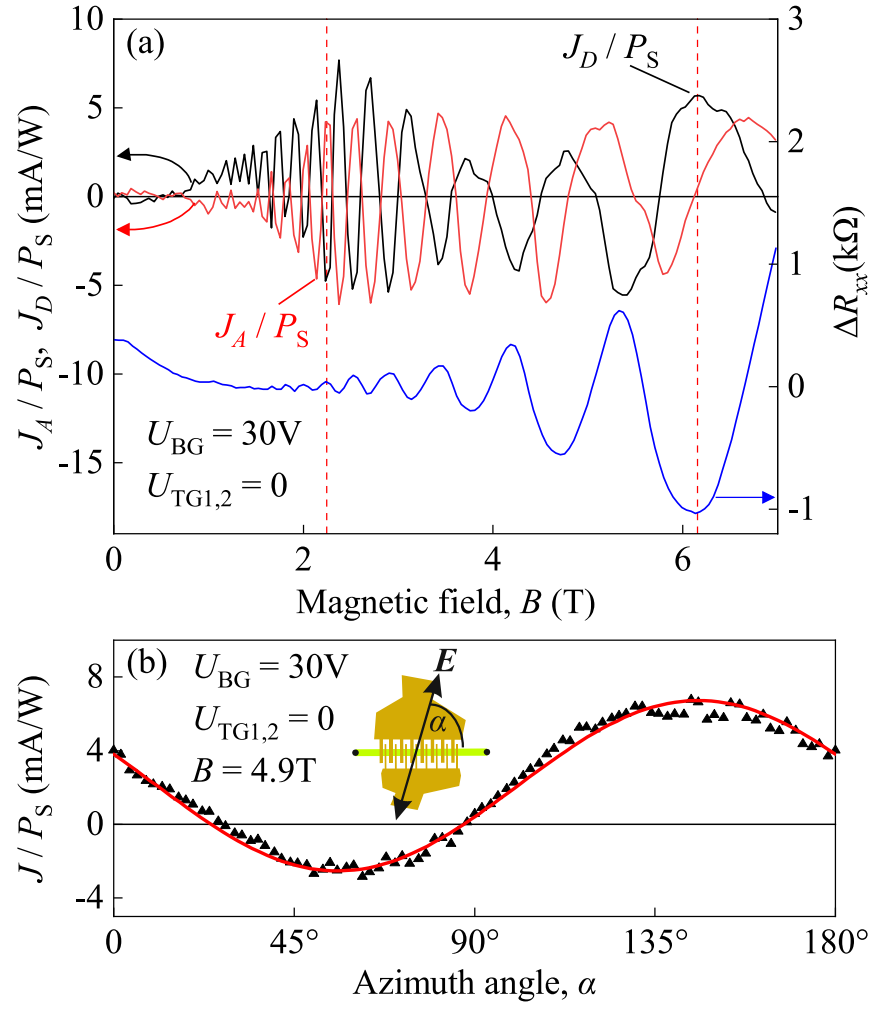

FIG. 6. (a) Magnetic field dependences of the ratchet photocurrent amplitudes $J_{A}$ and $J_{D}$ normalized by the radiation power coming onto the sample, $P_{\mathrm{S}}$. To extract $J_{A}$ and $J_{D}$ we measured magnetic field dependencies for two angles $\alpha=0$ and $90^{\circ}$, at which $P_{\mathrm{L}}= \pm 1$ and $\tilde{P}_{\mathrm{L}}=0$. Then, the curves were calculated after $J_{A}=[J(\alpha=0)-$ $\left.J\left(\alpha=90^{\circ}\right)\right] / 2$ and $J_{D}=\left[J(\alpha=0)+J\left(\alpha=90^{\circ}\right)\right] / 2$, where $J(\alpha=$ $0)$ and $J\left(\alpha=90^{\circ}\right)$ are photocurrent measured for radiation electric field vector oriented $\boldsymbol{E}$ perpendicular and parallel to the DGG stripes, respectively. (b) Dependence of the ratchet photocurrent normalized by the radiation coming onto the sample, $P_{\mathrm{S}}$, on the azimuth angle $\alpha$ obtained for a magnetic field of $B=4.9 \mathrm{~T}$. Red line shows fit according to Eq. (4) with fitting parameters $J_{A} / P_{\mathrm{S}}=17 \mathrm{~mA} / \mathrm{W}$, $J_{B} / P_{\mathrm{S}}=-4.3 \mathrm{~mA} / \mathrm{W}$, and $J_{D} / P_{\mathrm{S}}=2.1 \mathrm{~mA} / \mathrm{W}$. Inset shows experimental setup and defines angle $\alpha$ describing relative orientation of the radiation electric field vector $\boldsymbol{E}$ and DGG structure.

reveals that these ratchet current contributions have opposite signs and close magnitudes.

Above we discussed experiments with linear polarization rotated by $\lambda / 2$ plate. Let us now discuss experimental data obtained by using $\lambda / 4$ plate which allows us to create circular polarization. Figure 7(a) shows magneto-oscillations of the photocurrent excited by right- $\left(\sigma^{+}\right)$and left-handed $\left(\sigma^{-}\right)$ circularly polarized radiation. Subtracting these two curves we obtain the amplitude of the helicity-sensitive photocurrent $J_{C}$ given by

$$
J_{C}=\frac{J\left(\sigma^{+}\right)-J\left(\sigma^{-}\right)}{2} .
$$

This treatment extracts the photocurrent contribution whose direction reverses upon switching the radiation helicity. Note that for circularly polarized radiation the degrees of linear polarization $P_{\mathrm{L}}$ and $\tilde{P}_{\mathrm{L}}$ are equal to zero and, consequently, the magnetic ratchet effect caused by the linearly polarized 

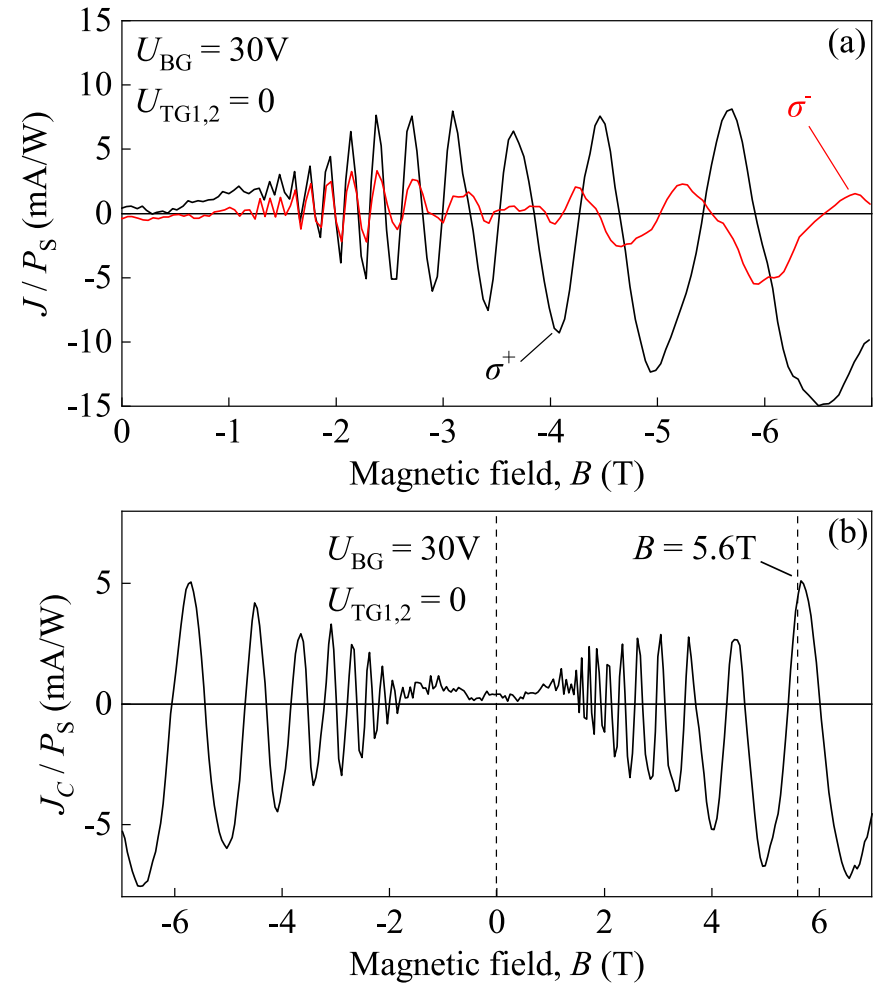

FIG. 7. (a) Dependences of the normalized ratchet photocurrent induced by right-handed (black curve) and left-handed (red curve) circularly polarized radiation on the magnetic field $B$. (b) Magnetic field dependence of the amplitude of the helicity-dependent ratchet current $J_{C}$ normalized by the radiation power $P_{S}$. $J_{C}$ was calculated according to Eq. (5).

radiation vanishes. This sign-reversion has been observed directly by measuring the dependence of the photocurrent on the phase angle $\varphi$ defining the radiation helicity after Eq. (3). This is shown in Fig. 8(a), where the helicity dependence of the ratchet photocurrent is studied at $B=5.6 \mathrm{~T}$, corresponding to the maximum of $J_{C}$; see Fig. 7(b). The overall polarization

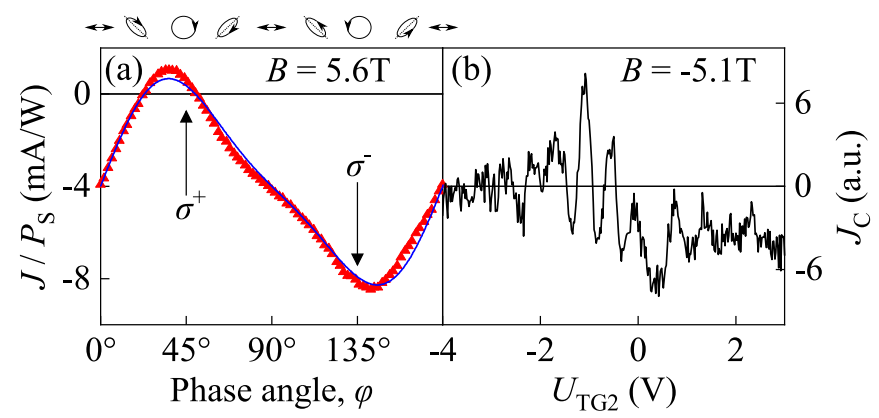

FIG. 8. (a) Dependence of the ratchet photocurrent on the radiation helicity for a magnetic field of $B=5.6 \mathrm{~T}$. Arrows above the plot illustrate the polarization state. Blue line shows fit according to Eq. (6) with fitting parameters $J_{A} / P_{\mathrm{S}}=-0.2 \mathrm{~mA} / \mathrm{W}, J_{B} / P_{\mathrm{S}}=$ $1.8 \mathrm{~mA} / \mathrm{W}, J_{C} / P_{\mathrm{S}}=4.1 \mathrm{~mA} / \mathrm{W}$, and $J_{D} / P_{\mathrm{S}}=-3.8 \mathrm{~mA} / \mathrm{W}$. (b) Dependence of the amplitude of the helicity-dependent ratchet current $J_{C}$ on the second top gate voltage at $B=-5.1 \mathrm{~T}$. dependence of the photocurrent can be well fitted by

$$
J=J_{A} P_{\mathrm{L}}(\varphi)+J_{B} \tilde{P}_{\mathrm{L}}(\varphi)+J_{C} P_{\text {circ }}(\varphi)+J_{D},
$$

where $J_{A}, J_{B}, J_{C}$, and $J_{D}$ are magnetic field-dependent fitting parameters. Figure 8(a) demonstrates that the circular photocurrent yields substantial contribution to the total photocurrent. Similar to the linear ratchet effect the circular photocurrent shows clear oscillations upon variation of the gate potential; see Fig. 8(b) for $J_{C}\left(U_{\mathrm{TG} 2}\right)$.

To summarize the experimental results, we observed that excitation of the DGG superlattice with $\mathrm{THz}$ radiation results in the ratchet photocurrent showing magneto-oscillations as well as oscillations upon variation of back or top gate voltages. The oscillations are closely related to the $\mathrm{SdH}$ effect. Measurements with controllable variation of the top gate voltages and, correspondingly, the lateral asymmetry parameter $\Xi$ clearly demonstrate that the photocurrent is caused by the ratchet effect. The photocurrent is giantly enhanced in the presence of magnetic field. The experimental results show a substantial contribution of both, linear and circular magnetic ratchet effects exhibiting sign-alternating magnetooscillations.

\section{THEORY}

In this section we generalize the theory of magnetic ratchets to graphene-based systems in the $\mathrm{SdH}$ regime. While for ratchets based on two-dimensional systems with a parabolic energy spectrum the theory of magneto-oscillations was developed in Refs. [46-48] it can not be applied to graphene. Moreover, as it has been demonstrated in Ref. [28], even at zero magnetic field the ratchet currents are drastically different in systems with linear and parabolic energy dispersions.

We use the Boltzmann kinetic equation approach, where the electric current density is given by the following expression:

$$
\boldsymbol{j}=e \sum_{v, p} \boldsymbol{v}_{\boldsymbol{p}} \bar{f}_{\boldsymbol{p}}
$$

Here $\boldsymbol{v}_{p}=v_{0} \boldsymbol{p} / p$ is the velocity of carriers having the momentum $\boldsymbol{p}$ with $v_{0}$ being the Dirac fermion velocity, $v$ enumerates spin and valley-degenerate states, and $\bar{f}_{p}$ is the distribution function $f_{p}(x)$ averaged over the space period of DGG structure. The latter is a solution of the kinetic equation [46]

$$
\left(\frac{\partial}{\partial t}+v_{\boldsymbol{p}, x} \frac{\partial}{\partial x}+\boldsymbol{F}_{\boldsymbol{p}} \cdot \frac{\partial}{\partial \boldsymbol{p}}\right) f_{\boldsymbol{p}}(x)=\operatorname{St}\left[f_{\boldsymbol{p}}(x)\right] .
$$

Here $\operatorname{St}[f]$ is the elastic scattering collision integral, and the space- and time-dependent force is given by

$$
\boldsymbol{F}_{\boldsymbol{p}}=e \boldsymbol{E}(x) \mathrm{e}^{-i \omega t}+e \boldsymbol{E}^{*}(x) \mathrm{e}^{i \omega t}+\frac{e}{c} \boldsymbol{v}_{\boldsymbol{p}} \times \boldsymbol{B}-\frac{d V}{d x} \hat{\boldsymbol{x}},
$$

where $\boldsymbol{E}(x)$ is the radiation near-field acting on twodimensional carriers, $\omega$ is the radiation frequency, and $V(x)$ is the periodic potential of the ratchet. The distribution function is found by sequential iterations of the kinetic equation in small electric field amplitude and the ratchet potential with the result linear in $d V / d x$ and quadratic in $|\boldsymbol{E}(x)|$ with the ratchet 
current proportional to the asymmetry parameter $\Xi$ given by Eq. (1).

For zero magnetic field the polarization-dependent ratchet current density in graphene is given by [28,29]

$$
\begin{aligned}
& j_{x}^{0}=-j_{0} \frac{(\omega \tau)^{2}}{1+(\omega \tau)^{2}}\left[\frac{2(\omega \tau)^{2}}{1+(\omega \tau)^{2}}+P_{\mathrm{L}}\right], \\
& j_{y}^{0}=-j_{0} \frac{\omega \tau}{1+(\omega \tau)^{2}}\left[\omega \tau \tilde{P}_{\mathrm{L}}+\frac{(\omega \tau)^{2}-1}{1+(\omega \tau)^{2}} P_{\text {circ }}\right],
\end{aligned}
$$

where $j_{0}$ is

$$
j_{0}=\Xi \frac{e^{3} v_{0}^{2} \tau}{2 \pi \hbar^{2} \varepsilon_{\mathrm{F}} \omega^{2}},
$$

and $\tau$ is the electron elastic scattering time assumed to be independent of the Fermi energy.

Here we calculate the ratchet current in graphene in the $\mathrm{SdH}$ regime. The quantization of the energy spectrum in strong magnetic fields is taken into account by the oscillating density of states at the Fermi energy: $g=g_{0}\left(1+\delta_{c}\right)$, where $g_{0}=2 \varepsilon_{\mathrm{F}} /\left(\pi \hbar^{2} v_{0}^{2}\right)$ is the zero-field density of states with account for spin and valley degeneracies, and the oscillating part is given by $[49,50]$

$$
\delta_{c}=2 \cos \left(\pi \varepsilon_{\mathrm{F}} / \hbar \omega_{c}\right) \exp \left(-\pi / \omega_{c} \tau_{q}\right) .
$$

Here the cyclotron frequency in graphene is $\omega_{c}=e B v_{0}^{2} / \varepsilon_{\mathrm{F}}$, and $\tau_{q}$ is the quantum lifetime. As a result of the densityof-states oscillations, the electron scattering rate also has an oscillating part: $\gamma=\tau^{-1}\left(1+\delta_{c}\right)$.

In the Appendix, we find the distribution function $\bar{f}_{p}(x)$. Note that not only the angular-independent part of the distribution function but also its second angular harmonics contribute to the current Eq. (7) in graphene due to nonparabolicity of the Dirac fermion dispersion [28]. Averaging the product $\boldsymbol{v}_{\boldsymbol{p}} \bar{f}_{\boldsymbol{p}}(x)$ over directions of $\boldsymbol{p}$ and then, integrating by the electron energy, taking into account the oscillations of the density of states, we obtain the ratchet current components in the form given in the Appendix, Eq. (A41). In general, we obtain that the leading contribution to the magnetic ratchet current is proportional to $\partial^{2} \delta_{c} / \partial \varepsilon_{\mathrm{F}}^{2} \approx-\left(2 \pi / \hbar \omega_{c}\right)^{2} \delta_{c}$. This yields $1 / B$ oscillations which are in phase with the $\mathrm{SdH}$ oscillations. Note that there is also a contribution proportional to the first derivative of $R_{x x}$ with respect to $B\left(j \propto d R_{x x} / d B\right)$ phase shifted by $\pi / 2$ from $R_{x x}(B)$. However, this contribution is small with respect to the quantum parameter $\hbar \omega_{c} /\left(2 \pi \varepsilon_{\mathrm{F}}\right) \ll$ 1 , and, consequently, we omit it in the following calculations.

The obtained expressions, valid for arbitrary relation between the parameters $\omega_{c}, \omega$, and $\tau$, are cumbersome, therefore we give them here in two limits of low and high magnetic fields where $\omega_{c}$ is much higher and much smaller than $\omega$, respectively.

$$
\begin{aligned}
& \text { At } \begin{aligned}
1 / \tau & \left.\ll \omega_{c} \ll \omega \text { (i.e., } B \ll B_{C R}\right) \text { we have } \\
j_{x}= & -\frac{j_{0}}{\left(\omega_{c} \tau\right)^{2}}\left(\frac{2 \pi \varepsilon_{\mathrm{F}}}{\hbar \omega_{c}}\right)^{2} \delta_{c} \\
\times & {\left[1-\frac{5}{8\left(\omega_{c} \tau\right)^{2}} P_{\mathrm{L}}+\frac{1}{4 \omega_{c} \tau} \tilde{P}_{\mathrm{L}}+\frac{3 \omega_{c}}{2 \omega} P_{\text {circ }}\right], }
\end{aligned}
\end{aligned}
$$

$$
\begin{aligned}
j_{y}= & \frac{j_{0}}{2\left(\omega_{c} \tau\right)^{2}}\left(\frac{2 \pi \varepsilon_{\mathrm{F}}}{\hbar \omega_{c}}\right)^{2} \delta_{c} \\
& \times\left[\frac{4}{\omega_{c} \tau}+\frac{1}{2 \omega_{c} \tau} P_{\mathrm{L}}+\frac{5}{4\left(\omega_{c} \tau\right)^{2}} \tilde{P}_{\mathrm{L}}+\frac{5}{\omega \tau} P_{\text {circ }}\right] .
\end{aligned}
$$

In the opposite limit $1 / \tau \ll \omega \ll \omega_{c}\left(B \gg B_{C R}\right)$ we get

$$
\begin{aligned}
j_{x}= & -\frac{j_{0} \omega^{2}}{4 \omega_{c}^{4} \tau^{2}}\left(\frac{2 \pi \varepsilon_{\mathrm{F}}}{\hbar \omega_{c}}\right)^{2} \delta_{c} \\
& \times\left[-1-\frac{7}{2\left(\omega_{c} \tau\right)^{2}} P_{\mathrm{L}}+\frac{3}{\omega_{c} \tau} \tilde{P}_{\mathrm{L}}+\frac{3 \omega}{2 \omega_{c}} P_{\text {circ }}\right], \\
j_{y}= & \frac{j_{0} \omega^{2}}{2 \omega_{c}^{4} \tau^{2}}\left(\frac{2 \pi \varepsilon_{\mathrm{F}}}{\hbar \omega_{c}}\right)^{2} \delta_{c} \\
& \times\left[-\frac{1}{4 \omega_{c} \tau}+\frac{3}{2 \omega_{c} \tau} P_{\mathrm{L}}+\frac{7}{4\left(\omega_{c} \tau\right)^{2}} \tilde{P}_{\mathrm{L}}+\frac{1}{\omega \tau} P_{\text {circ }}\right] .
\end{aligned}
$$

Here $j_{0}$ is the zero-field value of the ratchet current, Eq. (11).

Above we developed the drift-diffusion theory assuming that the impurity scattering dominates over the electronelectron scattering. The discussion of the hydrodynamic regime, where electron-electron collisions are very fast, will be presented elsewhere (previous studies on the hydrodynamic ratchet effect [30-32,51] did not discuss magnetooscillations). Our preliminary estimates show that similar results can be obtained. In particular, we find that in the high-field limit $\omega_{c} \gg \omega$ at $\boldsymbol{E} \| x$ the ratio $j_{x} / j_{y} \sim \omega_{c} \tau$ in accordance with Eqs. (15) and (16).

\section{DISCUSSION}

Now we discuss the experimental results in the view of the developed theory. In the experiments, we probed the photocurrent flowing in the $x$ direction normal to the DGG stripes, because of the device geometry. The photocurrent obtained in Sec. IV is proportional to $\delta_{c}$, i.e., exhibits $1 / B$ periodic oscillations following $\mathrm{SdH}$ oscillations. Analyzing the extrema positions of the photocurrent (see Fig. 3), we obtained that the oscillations indeed correspond to the $\mathrm{SdH}$ oscillations of $R_{x x}(B)$, i.e., ratchet photocurrent $J$ is proportional to $\delta_{c}$ as expected from Eqs. (13) and (15). We note that at high magnetic fields the experimentally observed ratchet current oscillations have a magnetic field-dependent phase shift, and, hence, the photocurrent is phase-shifted in respect to the oscillations of $R_{x x}(B)$. Exploring this exciting feature requires additional experimental and theoretical studies, and is out of scope of this paper.

From Eqs. (13) and (15) together with Eq. (12) we expect sign-alternating oscillations of the magnetic ratchet current as a function of Fermi energy and, consequently, gate voltages. The oscillations periodic in gate voltage are indeed observed at a fixed magnetic field for back- as well as top gates; see Figs. 4 and 5, respectively. Comparing Figs. 4 and 5 we see a substantial difference in the period of oscillations. This is just caused by the different thicknesses (capacities) of the corresponding insulator layers.

As a fingerprint of the ratchet effect, the magnetophotocurrent $j_{x} \propto j_{0}$ is proportional to the lateral asymmetry 


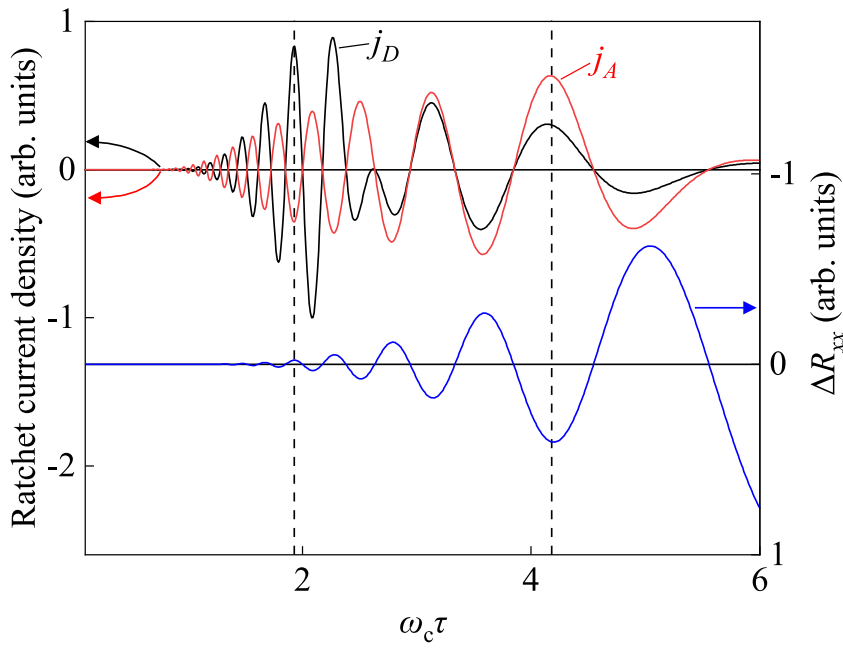

FIG. 9. Theoretical dependences of the magnetic ratchet photocurrents on $\omega_{c} \tau \propto B$. Black curve shows the polarization independent contribution $j_{D}$ ( $J_{D}$ in experiment), red curve is the $P_{\mathrm{L}}$-linear contribution $j_{A}\left(J_{A}\right.$ in experiment). The photocurrents are calculated by using Eq. (A41) at $\omega \tau=4.6, \tau_{q} / \tau=0.3, \varepsilon_{\mathrm{F}} \tau / \hbar=25$.

parameter $\Xi$; see Eqs. (1) and (10). The latter can easily be varied by the variation of the top gate polarities and relative amplitudes. This is indeed observed in experiment; see Fig. 5, which shows that for large top gate voltages the sign of oscillations is opposite for opposite signs of the parameter $\Xi$; see the black and red vertical arrows in Fig. 5. Note that for both top gate voltages equal to zero the magnetic ratchet currents are caused by the built-in asymmetry; see Fig. 5 .

The developed theory also demonstrates that, as observed in the experiment, the magneto-oscillations of the ratchet current are highly sensitive to the polarization state of the incident radiation. For linearly polarized radiation the magnetic ratchet current consists of the polarization-independent current as well as of two contributions varying upon rotation of the radiation polarization plane as $P_{\mathrm{L}}=\cos 2 \alpha$ and $\tilde{P}_{\mathrm{L}}=\sin 2 \alpha$; see Eqs. (13) and (15). These contributions are clearly detected in experiment (see Fig. 6) and exhibit signalternating magneto-oscillations. The experimental results for the corresponding factors $J_{D}$ and $J_{A}$, yielding dominating contributions for wide range of magnetic fields, are presented in Figs. 3 and 6(a). Note that the photocurrent shown in the latter figure is obtained for $\alpha=0$ and presents the sum of $J_{A}$ and $J_{D}$.

The theory also describes well the dependences of the oscillation amplitudes on the magnetic field. Figure 9 shows calculated magnetic field dependences of the coefficients $j_{D}$ and $j_{A}$ describing polarization-independent magnetic ratchet photocurrent density $j_{D}$, and the one driven by linearly polarized radiation, $j_{A} P_{\mathrm{L}}$. Note that the coefficients $j_{D}$ and $j_{A}$ are introduced in the same way as used in the experimental fit function Eq. (4) having current contributions $J_{D}$ and $J_{A} P_{\mathrm{L}}(\alpha)$. The overall behavior of the oscillations is the same: an increase of magnetic field first enormously magnifies the oscillations magnitude, which, however, decreases for further magnetic field increase. Analytically, this nonmonotonous behavior, which is absent in $\mathrm{SdH}$ oscillations, is described by the ratio of the current oscillation amplitude $j^{*}$ introduced according to $j_{D}=j^{*} \cos \left(\pi \varepsilon_{\mathrm{F}} / \hbar \omega_{c}\right)$ to that of the zero field current $j_{0}$. Then, for the polarization independent contribution, we obtain from Eq. (13)

$$
\frac{j^{*}}{j_{0}} \sim\left(\frac{2 \pi \varepsilon_{\mathrm{F}}}{\hbar \omega_{c}}\right)^{2} \frac{\exp \left(-\pi / \omega_{c} \tau_{q}\right)}{\left(\omega_{c} \tau\right)^{2}} .
$$

A giant increase of the ratchet current when applying a magnetic field is caused by the first factor since $\varepsilon_{F} / \hbar \omega_{c} \gg 1$ so that $j^{*}>j_{0}$ up to $\omega_{c} \tau \approx 9$, whereas for $\omega_{c} \tau \lesssim 2.2$ the amplitude $j^{*}$ rises due to factor $\exp \left(-\pi / \omega_{c} \tau_{q}\right)$ and $j^{*} / j_{0} \gg$ 1 , a further increase in magnetic field leads to a decrease in the ratio. This is caused by the competition of the exponential factor, saturating at $\omega_{c} \tau_{q} \gg 1$, with the factor $\omega_{c}^{-4}$. This results in a maximum of the current (see Fig. 9) clearly detected in experiment; see Fig. 6(a). Note that a possible contribution of the Seebeck ratchet effect in quantizing magnetic fields $[46,47]$ can contribute to the magnitude of the polarizationindependent current.

Similar analysis of the magnetic field dependence can also be performed for the linear-polarization driven photocurrent $j_{A}$. From Eq. (13) follows that, alike $j_{D}$, the photocurrent $j_{A}$ drastically increases with the magnetic field increase, reaches maximum and decreases at further magnetic field increase. The only difference between the amplitudes $j_{A}$ and $j_{D}$ is that in a high fields $j_{A}$ decreases faster, as $\omega_{c}^{-6}$. Figure 9 shows that, for the magnetic field relevant to experiments, both amplitudes, $j_{A}$ and $j_{D}$, yield comparable contributions. This agrees with experiment; see Fig. 6(a). Furthermore, from Eq. (13) and Fig. 9 it follows that, at low magnetic fields, the polarization-independent component amplitude $j_{D}$ and that for the current sensitive to the orientation of the radiation electric field vector $j_{A}$ have opposite signs. This is in a fully agreement with experiment; see Fig. 6(a) for $B \leqslant 2.5$ T. Note that more detailed comparison of the theory and experiments is complicated by the magnetic field-dependent phase shift addressed above as well as by a possible contribution of plasmonic effects [30-32,51].

Besides the photocurrent sensitive to the degree of linear polarization, experiments show a substantial input of the magnetic ratchet current $j_{x} \propto P_{\text {circ }}$, which changes its sign upon switching the radiation helicity. Figures 7 and 8(a) show magneto-oscillations of this current, and its dependence on the phase angle $\varphi$. The helicity-driven contribution is also expected from the developed theory; see the last terms in square brackets on the right sides of Eqs. (13) and (15). For circularly polarized radiation, the photocurrents proportional to $P_{\mathrm{L}}$ and $\tilde{P}_{\mathrm{L}}$ vanish, and the total ratchet current for high magnetic fields is given by $j_{x} \propto 1-\left(3 \omega / 2 \omega_{c}\right) P_{\text {circ }}$. For magnetic fields $B>4 \mathrm{~T}$ and $f=2.54 \mathrm{THz}$ we obtain that the amplitudes of the helicity-dependent and polarization-independent currents are comparable, which is in agreement with experiment; see Fig. 8(a). Note that, in agreement with Eq. (15), these currents have opposite signs. Similarly to the magnetic ratchet current driven by linearly polarized radiation, oscillations are expected as a function of top gate voltage and, indeed detected in the experiment; see Fig. 8(b).

Finally, we note that we do not aim here at studying in detail the ratchet effect in the vicinity of the cyclotron resonance, 
which for a systematic study would require using radiation of different frequencies. However, our preliminary theoretical estimates show that the ratchet current behavior can be quite interesting. In particular, in the vicinity of the cyclotron resonance the maximum difference between the drift-diffusion regime, studied in this work, and the hydrodynamic regime is expected. This interesting issue deserves a future publication. Therefore, above we give theoretical formulas only for the cases of high and low fields as compared with the resonance field.

\section{SUMMARY}

Our experiments together with the developed theory show that terahertz radiation applied to graphene with asymmetric, lateral superlattice and subjected to a strong magnetic field promotes the magnetic quantum ratchet effect. The characteristic feature of the magnetic field induced ratchet current is magneto-oscillations with a magnitude much larger than the ratchet current at zero magnetic field. This, caused by Shubnikov-de Haas effect, enhancement of the ratchet effect is insofar generic as it is not only observed in graphene superlattices, but also in quantum well structures with parabolic spectrum $[47,48]$. The amplitude and direction of the ratchet current are controlled by the lateral asymmetry parameter $\Xi$, magnetic field strength/direction, and the radiation's polarization state. The latter reflects magnetic ratchet current contributions driven by linearly and circularly polarized radiation. The presented theory describes well almost all results. It cannot, however, explain the magnetic field-dependent phase shift of the ratchet current oscillations observed at high magnetic fields. This striking result may be caused by contributions from plasmonic ratchets [32], neglected here. Its understanding is a future task.

\section{ACKNOWLEDGMENTS}

The support from the FLAG-ERA program (project DeMeGRaS, Deutsche Forschungsgemeinschaft (DFG) No. GA 501/16-1) and the Volkswagen Stiftung Program (97738) is gratefully acknowledged. The work of L.E.G. and V.Yu.K. was supported by the Foundation for the Advancement of Theoretical Physics and Mathematics "BASIS." L.E.G. also thanks the Russian Foundation for Basic Research (project 19-02-00095). V.Yu.K. also thanks the Russian Foundation for Basic Research (Grant No. 20-02-00490). S.D.G. and V.Yu.K. thank the Foundation for Polish Science (IRA Program, grant MAB/2018/9, CENTERA) for support.

\section{APPENDIX: DERIVATION OF THE RATCHET CURRENT}

The ratchet current Eq. (15) is obtained by sequential iterations of the kinetic equation (8) at two small perturbations, namely the light amplitude $\boldsymbol{E}$ and the periodic ratchet potential $V(x)$. The first iteration step is always account for $\boldsymbol{E}$, but the next steps could be different. One contribution is obtained if the potential $V$ is taken into account at the second stage, and the radiation amplitude $\boldsymbol{E}$ at the last stage. We denote the corresponding correction to the distribution function $f^{(E V E)}$.
In contrast to systems with parabolic energy dispersion, the total ratchet current is not restricted to this contribution. An additional contribution to the ratchet current, $\delta \boldsymbol{j}$, is obtained if the amplitude $\boldsymbol{E}$ is taken into account twice assuming $V=0$, and then, at the last stage, the periodic potential is taken into account. The corresponding part of the distribution function is denoted as $f^{(E E V)}$. In the next two subsections we derive both contributions to the ratchet current.

\section{EVE contribution}

The distribution function $f^{(E V E)}$ is a solution of the kinetic equation bilinear in $\boldsymbol{E}$ and linear in $V(x)$ obtained by a simultaneous account for $\boldsymbol{E}, V(x)$, and then $\boldsymbol{E}$.

The kinetic equation for $f^{(E V E)}$ has the form

$$
\omega_{c} \frac{\partial f^{(E V E)}}{\partial \varphi}+e \boldsymbol{E}^{*} \cdot \frac{\partial f^{(E V)}}{\partial \boldsymbol{p}}=-\gamma f^{(E V E)},
$$

where $f^{(E V)}$ is the correction bilinear in $\boldsymbol{E}$ and $V(x)$, and

$$
\gamma(\varepsilon)=\frac{1}{\tau}\left[1+\delta_{c}(\varepsilon)\right] .
$$

The solution is given by

$$
f^{(E V E)}=-\sum_{ \pm} \tau_{c}^{ \pm} e \boldsymbol{E}^{*} \cdot\left(\frac{\partial f^{(E V)}}{\partial \boldsymbol{p}}\right)_{ \pm}+\text {c.c. },
$$

where $(\ldots)_{ \pm}$denotes the \pm 1 st Fourier harmonics, and

$$
\tau_{c}^{ \pm}=\frac{1}{\gamma(\varepsilon) \pm i \omega_{c}} .
$$

Here $\delta_{c}(\varepsilon)$ means $\delta_{c}$ given by Eq. (12) where $\varepsilon_{\mathrm{F}}$ is substituted by $\varepsilon$.

Substituting the solution (A3) into Eq. (7), we obtain the current density in the form

$$
j_{\alpha}=-e^{2} \sum_{\boldsymbol{p}} v_{\alpha} \sum_{ \pm} \tau_{c}^{ \pm} \boldsymbol{E}^{*} \cdot\left(\frac{\partial f^{(E V)}}{\partial \boldsymbol{p}}\right)_{ \pm}+\text {c.c. }
$$

This equation shows that only the even in $\boldsymbol{p}$ part of $f^{(E V)}$ contributes to the photocurrent. It contains two terms, the $\varphi$-independent one and the second harmonics of $\varphi$. For $j_{+}=$ $j_{x}+i j_{y}$ we get

$$
\begin{aligned}
j_{+}= & -e^{2} \sum_{\boldsymbol{p}} v_{+} \tau_{c}^{-} \boldsymbol{E}^{*} \cdot\left(\frac{\partial f^{(E V)}}{\partial \boldsymbol{p}}\right)_{-} \\
& +\left(\boldsymbol{E} \leftrightarrow \boldsymbol{E}^{*}, \omega \rightarrow-\omega\right) .
\end{aligned}
$$

Integrating by parts we obtain

$$
j_{+}=e^{2} \sum_{\boldsymbol{p}} \frac{\partial\left(v_{+} \tau_{c}^{-}\right)}{\partial \boldsymbol{p}} \cdot \boldsymbol{E}^{*} f^{(E V)}+\left(\boldsymbol{E} \leftrightarrow \boldsymbol{E}^{*}, \omega \rightarrow-\omega\right) .
$$

Calculating the gradient in the momentum space

$$
\frac{\partial\left(v_{+} \tau_{c}^{-}\right)}{\partial \boldsymbol{p}} \cdot \boldsymbol{E}^{*}=\frac{v_{0}^{2} \varepsilon}{2}\left(\frac{\tau_{c}^{-}}{\varepsilon}\right)^{\prime}\left[\left(E^{*}\right)_{+}+\left(E^{*}\right)_{-} \mathrm{e}^{2 i \varphi_{p}}\right],
$$


where $\left(E^{*}\right)_{ \pm}=E_{x}^{*} \pm i E_{y}^{*}$, and the prime denotes differentiating over $\varepsilon$, we obtain

$$
\begin{aligned}
j_{+}= & \frac{e^{2} v_{0}^{2}}{2} \sum_{\boldsymbol{p}} \varepsilon\left(\frac{\tau_{c}^{-}}{\varepsilon}\right)^{\prime}\left[\left(E^{*}\right)_{+} f_{0}^{(E V)}+\left(E^{*}\right)_{-} f_{-2}^{(E V)}\right] \\
& +\left(\boldsymbol{E} \leftrightarrow \boldsymbol{E}^{*}, \omega \rightarrow-\omega\right) .
\end{aligned}
$$

Here $f_{0,-2}^{(E V)}$ mean the angular-independent part of $f^{(E V)}$ and the part $\propto \mathrm{e}^{-2 i \varphi_{p}}$.

Since the angular integration is already performed, we can pass from summation over $\boldsymbol{p}$ to integration over energy:

$$
\begin{aligned}
j_{+}= & \frac{e^{2} v_{0}^{2}}{2} \int d \varepsilon g(\varepsilon) \varepsilon\left(\frac{\tau_{c}^{-}}{\varepsilon}\right)^{\prime}\left[\left(E^{*}\right)_{+} f_{0}^{(E V)}+\left(E^{*}\right)_{-} f_{-2}^{(E V)}\right] \\
& +\left(\boldsymbol{E} \leftrightarrow \boldsymbol{E}^{*}, \omega \rightarrow-\omega\right),
\end{aligned}
$$

where $g(\varepsilon)$ is the density of states. The corrections $f_{0,-2}^{(E V)}$ are given by

$$
\begin{aligned}
& f_{0}^{(E V)}=\frac{i e v_{0}^{2}}{4 \omega} \sum_{ \pm}\left[-f_{0}^{\prime} E_{x} \frac{d V}{d x} \frac{\left(\varepsilon \tau_{1 \omega}^{ \pm}\right)^{\prime}}{\varepsilon}+V \frac{d E_{x}}{d x} \tau_{1 \omega}^{ \pm} f_{0}^{\prime \prime}\right], \\
& f_{-2}^{(E V)}=\frac{e v_{0}^{2} \tau_{2 \omega}^{-}}{4}\left[-f_{0}^{\prime} E_{+} \frac{d V}{d x} \varepsilon\left(\frac{\tau_{1 \omega}^{-}}{\varepsilon}\right)^{\prime}+V \frac{d E_{+}}{d x} \tau_{1 \omega}^{-} f_{0}^{\prime \prime}\right],
\end{aligned}
$$

where

$$
\tau_{n \omega}^{ \pm}=\frac{1}{\gamma(\varepsilon)-i \omega \pm i n \omega_{c}}, \quad n=1,2 .
$$

These expressions at $\omega_{c}=0$ pass into the corresponding expressions from Ref. [28].

Substituting $f_{0,-2}^{(E V)}$ into Eq. (A10), we obtain

$$
j_{+}=j_{+}^{(0)}+j_{+}^{(-2)}+\left(\boldsymbol{E} \leftrightarrow \boldsymbol{E}^{*}, \omega \rightarrow-\omega\right),
$$

where

$$
\begin{aligned}
j_{+}^{(0)}= & \frac{i e^{3} v_{0}^{4}}{8 \omega} \sum_{ \pm} \int d \varepsilon g(\varepsilon) \varepsilon\left(\frac{\tau_{c}^{-}}{\varepsilon}\right)^{\prime}\left(E^{*}\right)_{+} \\
& \times\left[-f_{0}^{\prime} E_{x} \frac{d V}{d x} \frac{\left(\varepsilon \tau_{1 \omega}^{ \pm}\right)^{\prime}}{\varepsilon}+V \frac{d E_{x}}{d x} \tau_{1 \omega}^{ \pm} f_{0}^{\prime \prime}\right], \\
j_{+}^{(-2)}= & \frac{e^{3} v_{0}^{4}}{8} \int d \varepsilon g(\varepsilon) \varepsilon \tau_{2 \omega}^{-}\left(\frac{\tau_{c}^{-}}{\varepsilon}\right)^{\prime}\left(E^{*}\right)_{-} \\
& \times\left[-f_{0}^{\prime} E_{+} \frac{d V}{d x} \varepsilon\left(\frac{\tau_{1 \omega}^{-}}{\varepsilon}\right)^{\prime}+V \frac{d E_{+}}{d x} \tau_{1 \omega}^{-} f_{0}^{\prime \prime}\right] .
\end{aligned}
$$

Averaging over the $x$ coordinate with $E_{0}$ being the near-field amplitude yields

$$
\overline{E_{0} V \frac{d E_{0}}{d x}}=\frac{1}{2} \overline{V \frac{d E_{0}^{2}}{d x}}=-\frac{1}{2} \overline{E_{0}^{2} \frac{d V}{d x}} \equiv-\frac{1}{2} \Xi,
$$

and integrating over $\varepsilon$ we get

$$
\begin{aligned}
j_{+}^{(0)}= & \Xi\left(\left|e_{x}\right|^{2}+i e_{x} e_{y}^{*}\right) \frac{i e^{3} v_{0}^{4}}{8 \omega} \sum_{ \pm}\left\{g\left(\frac{\tau_{c}^{-}}{\varepsilon_{\mathrm{F}}}\right)^{\prime}\left(\varepsilon_{\mathrm{F}} \tau_{1 \omega}^{ \pm}\right)^{\prime}\right. \\
& \left.-\frac{1}{2}\left[g \varepsilon_{\mathrm{F}}\left(\frac{\tau_{c}^{-}}{\varepsilon_{\mathrm{F}}}\right)^{\prime} \tau_{1 \omega}^{ \pm}\right]^{\prime}\right\},
\end{aligned}
$$

$$
\begin{aligned}
j_{+}^{(-2)}= & \Xi\left(1-P_{\text {circ }}\right) \frac{e^{3} v_{0}^{4}}{8}\left\{g \varepsilon_{\mathrm{F}}^{2} \tau_{2 \omega}^{-}\left(\frac{\tau_{c}^{-}}{\varepsilon_{\mathrm{F}}}\right)^{\prime}\left(\frac{\tau_{1 \omega}^{-}}{\varepsilon_{\mathrm{F}}}\right)^{\prime}\right. \\
& \left.-\frac{1}{2}\left[g \varepsilon_{\mathrm{F}} \tau_{2 \omega}^{-}\left(\frac{\tau_{c}^{-}}{\varepsilon_{\mathrm{F}}}\right)^{\prime} \tau_{1 \omega}^{-}\right]^{\prime}\right\} .
\end{aligned}
$$

Here the prime denotes differentiation over $\varepsilon_{\mathrm{F}}$.

Let us analyze the terms in the curly brackets. The maximal result comes from the second derivative $\left(\tau_{c}^{-}\right)^{\prime \prime}$, therefore, only the second terms in curly brackets are important. The terms with the first derivative have much smaller amplitude due to the factor $\hbar \omega_{c} /\left(2 \pi \varepsilon_{\mathrm{F}}\right) \ll 1$. The terms $\sim \delta_{c}^{2}$ are also omitted because they have an additional small factor $\exp \left(-\pi / \omega_{c} \tau_{q}\right) \ll 1$ and result in oscillations with double period not present in the experiment. As a result, we obtain

$$
\begin{gathered}
j_{+}^{(0)}=-\Xi\left(i\left|e_{x}\right|^{2}-e_{x} e_{y}^{*}\right) \frac{e^{3} v_{0}^{4} g}{8} T_{\omega}\left(\tau_{c}^{-}\right)^{\prime \prime}, \\
j_{+}^{(-2)}=-\Xi\left(1-P_{\text {circ }}\right) \frac{e^{3} v_{0}^{4} g}{16} Q_{\omega}\left(\tau_{c}^{-}\right)^{\prime \prime},
\end{gathered}
$$

where $g=2 \varepsilon_{\mathrm{F}} /\left(\pi \hbar^{2} v_{0}^{2}\right)$ is the zero-field density of states (spin and valley degeneracies are taken into account), and

$$
T_{\omega}=\frac{\tau_{1 \omega}^{+}+\tau_{1 \omega}^{-}}{2 \omega}, \quad Q_{\omega}=\tau_{2 \omega}^{-} \tau_{1 \omega}^{-} .
$$

Finally, from Eq. (A12) we obtain the total current

$$
\begin{aligned}
j_{+}= & -\Xi \frac{e^{3} v_{0}^{2} \varepsilon_{\mathrm{F}}}{4 \pi \hbar^{2}}\left(\tau_{c}^{-}\right)^{\prime \prime} \\
& \times\left[Q_{+}+i T_{+}\left(1+P_{\mathrm{L}}+i \tilde{P}_{\mathrm{L}}\right)+\left(i T_{-}-Q_{-}\right) P_{\text {circ }}\right] .
\end{aligned}
$$

Here $T_{ \pm}=\left(T_{\omega} \pm T_{-\omega}\right) / 2, Q_{ \pm}=\left(Q_{\omega} \pm Q_{-\omega}\right) / 2$.

\section{EEV contribution}

Here we calculate the correction $\delta \boldsymbol{j}$ obtained by twice account for $\boldsymbol{E}$ and then for $V(x)$. The corresponding ratchet current is given by

$$
\delta j_{\alpha}=e \sum_{p} v_{\alpha} f^{(E E V)} .
$$

The kinetic equation for $f^{(E E V)}$ has the form

$$
\omega_{c} \frac{\partial f^{(E E V)}}{\partial \varphi}-\frac{d V}{d x} \frac{\partial f^{(E E)}}{\partial p_{x}}=-\gamma f^{(E E V)},
$$

where $f^{(E E)}$ is the correction bilinear in $\boldsymbol{E}$. The solution is given by

$$
f^{(E E V)}=\sum_{ \pm} \tau_{c}^{ \pm} \frac{d V}{d x}\left(\frac{\partial f^{(E E)}}{\partial p_{x}}\right)_{ \pm} .
$$

Substituting the solution (A24) into Eq. (A22), we obtain the current density in the form

$$
\delta j_{\alpha}=e \frac{d V}{d x} \sum_{p} v_{\alpha} \sum_{ \pm} \tau_{c}^{ \pm}\left(\frac{\partial f^{(E E)}}{\partial p_{x}}\right)_{ \pm} .
$$


For $\delta j_{+}=\delta j_{x}+i \delta j_{y}$ we get integrating by parts

$$
\delta j_{+}=-e \frac{d V}{d x} \sum_{p} \frac{\partial\left(v_{+} \tau_{c}^{-}\right)}{\partial p_{x}} f^{(E E)} .
$$

Calculating the derivative

$$
\frac{\partial\left(v_{+} \tau_{c}^{-}\right)}{\partial p_{x}}=\frac{v_{0}^{2} \varepsilon}{2}\left(\frac{\tau_{c}^{-}}{\varepsilon}\right)^{\prime}\left(1+\mathrm{e}^{2 i \varphi_{p}}\right),
$$

we obtain

$$
\delta j_{+}=-\frac{e v_{0}^{2}}{2} \frac{d V}{d x} \sum_{p} \varepsilon\left(\frac{\tau_{c}^{-}}{\varepsilon}\right)^{\prime}\left(f_{0}^{(E E)}+f_{-2}^{(E E)}\right) .
$$

Here $f_{0,-2}^{(E E)}$ mean the angular-independent part of $f^{(E E)}$ and the part $\propto \mathrm{e}^{-2 i \varphi_{p}}$. The former is controlled by energy relaxation processes: $f_{0}^{(E E)} \propto \tau_{\varepsilon}$ with $\tau_{\varepsilon}$ being the energy relaxation time. It describes the Seebeck and Nernst-Ettingshausen ratchet effects [46]. In what follows we omit this contribution concentrating on polarization-dependent ratchet currents.

Since the angular integration is already performed, we can pass from summation over $\boldsymbol{p}$ to integration over energy:

$$
\delta j_{+}=-\frac{e v_{0}^{2}}{2} \frac{d V}{d x} \int d \varepsilon g(\varepsilon) \varepsilon\left(\frac{\tau_{c}^{-}}{\varepsilon}\right)^{\prime} f_{-2}^{(E E)},
$$

where $g(\varepsilon)$ is the density of states. The correction $f_{-2}^{(E E)}$ is multiplied by $d V / d x$, therefore we find it in the quasihomogeneous limit:

$$
-2 i \omega_{c} f_{-2}^{(E E)}+e \boldsymbol{E}^{*} \cdot\left(\frac{\partial f^{(E)}}{\partial \boldsymbol{p}}\right)_{-2}=-\gamma f_{-2}^{(E E)},
$$

where the linear in $\boldsymbol{E}$ correction to the distribution function is found from

$$
\omega_{c} \frac{\partial f^{(E)}}{\partial \varphi}+e \boldsymbol{E} \cdot \boldsymbol{v} f_{0}^{\prime}=-\frac{f^{(E)}}{\tau_{1 \omega}} .
$$

The solutions are

$$
\begin{gathered}
f^{(E)}=-\frac{e v_{0}}{2} \sum_{ \pm} f_{0}^{\prime} \tau_{1 \omega}^{ \pm} \frac{p_{ \pm}}{p} E_{\mp}, \\
f_{-2}^{(E E)}=-\tau_{c 2} e \boldsymbol{E}^{*} \cdot\left(\frac{\partial f^{(E)}}{\partial \boldsymbol{p}}\right)_{-2}+\left(\boldsymbol{E} \leftrightarrow \boldsymbol{E}^{*}, \omega \rightarrow-\omega\right),
\end{gathered}
$$

where $\tau_{1 \omega}^{ \pm}$is given by Eq. (A11), and

$$
\tau_{c 2}=\frac{1}{\gamma(\varepsilon)-2 i \omega_{c}} .
$$

The calculation is performed as follows:

$$
\boldsymbol{E}^{*} \cdot\left(\frac{\partial}{\partial \boldsymbol{p}} f_{0}^{\prime} \tau_{1 \omega}^{ \pm} \frac{p_{ \pm} E_{\mp}}{p}\right)_{-2}=|E|^{2}\left(P_{\mathrm{L}}+i \tilde{P}_{\mathrm{L}}\right) \frac{v_{0}}{2} \varepsilon\left(\frac{\tau_{1 \omega}^{-} f_{0}^{\prime}}{\varepsilon}\right)^{\prime},
$$

which yields

$$
f_{-2}^{(E E)}=\frac{e^{2} \tau_{c 2} v_{0}^{2}}{4}|E|^{2}\left(P_{\mathrm{L}}+i \tilde{P}_{\mathrm{L}}\right) \varepsilon\left[\frac{\left(\tau_{1 \omega}^{-}+\tau_{1,-\omega}^{-}\right) f_{0}^{\prime}}{\varepsilon}\right]^{\prime} .
$$

Substituting $f_{-2}^{(E E)}$ into Eq. (A29) and averaging over the $x$ coordinate, we obtain

$$
\begin{aligned}
\delta j_{+}= & -\frac{e^{3} v_{0}^{4}}{4} \Xi\left(P_{\mathrm{L}}+i \tilde{P}_{\mathrm{L}}\right) \int d \varepsilon g(\varepsilon) \varepsilon^{2}\left(\frac{\tau_{c}^{-}}{\varepsilon}\right)^{\prime} \tau_{c 2} \\
& \times\left[\frac{\left(\tau_{1 \omega}^{-}+\tau_{1,-\omega}^{-}\right)}{2} \frac{f_{0}^{\prime}}{\varepsilon}\right]^{\prime} .
\end{aligned}
$$

Integrating over $\varepsilon$ we get

$$
\delta j_{+}=-\frac{e^{3} v_{0}^{4}}{4} \Xi\left(P_{\mathrm{L}}+i \tilde{P}_{\mathrm{L}}\right)\left[g \varepsilon_{\mathrm{F}}^{2}\left(\frac{\tau_{c}^{-}}{\varepsilon_{\mathrm{F}}}\right)^{\prime} \tau_{c 2}\right]^{\prime} \frac{\tau_{1 \omega}^{-}+\tau_{1,-\omega}^{-}}{2 \varepsilon_{\mathrm{F}}} .
$$

According to the same arguments as at calculation of the $E E V$ contribution (see the previous susbsection), the maximal result comes from $\left(\tau_{c}^{-}\right)^{\prime \prime}$ :

$$
\delta j_{+}=-\Xi \frac{e^{3} v_{0}^{2} \varepsilon_{\mathrm{F}}}{4 \pi \hbar^{2}}\left(\tau_{c}^{-}\right)^{\prime \prime} R\left(P_{\mathrm{L}}+i \tilde{P}_{\mathrm{L}}\right) .
$$

Here

$$
R=\tau_{c 2}\left(\tau_{1 \omega}^{-}+\tau_{1,-\omega}^{-}\right)
$$

\section{Total ratchet current}

A sum of $j_{+}$from Eq. (A21) and $\delta j_{+}$from Eq. (A39) yields the total ratchet current in the form

$$
\begin{aligned}
j_{x}+i j_{y}=- & \Xi \frac{e^{3} v_{0}^{2} \varepsilon_{\mathrm{F}}}{4 \pi \hbar^{2}}\left(\tau_{c}^{-}\right)^{\prime \prime}\left[Q_{+}+i T_{+}\right. \\
& \left.+\left(i T_{+}+R\right)\left(P_{\mathrm{L}}+i \tilde{P}_{\mathrm{L}}\right)+\left(i T_{-}-Q_{-}\right) P_{\text {circ }}\right] .
\end{aligned}
$$

Substituting

$$
\left(\tau_{c}^{-}\right)^{\prime \prime}=\delta_{c}\left(\frac{2 \pi}{\hbar \omega_{c}}\right)^{2} \frac{\tau}{\left(1-i \omega_{c} \tau\right)^{2}},
$$

and finding real and imaginary parts of Eq. (A41), we obtain the components of the total ratchet current.

For two limiting cases of low and high magnetic fields, passing to the limits $\omega \tau \gg \omega_{c} \tau \gg 1$ and $\omega_{c} \tau \gg \omega \tau \gg 1$ we get Eqs. (13) and (15), respectively.
[1] L. Vicarelli, M. S. Vitiello, D. Coquillat, A. Lombardo, A. C. Ferrari, W. Knap, M. Polini, V. Pellegrini, and A. Tredicucci, Nat. Mater. 11, 865 (2012).
[2] T. Otsuji, S. A. B. Tombet, A. Satou, H. Fukidome, M. Suemitsu, E. Sano, V. Popov, M. Ryzhii, and V. Ryzhii, J. Phys. D: Appl. Phys. 45, 303001 (2012). 
[3] M. Freitag, T. Low, W. Zhu, H. Yan, F. Xia, and P. Avouris, Nat. Commun. 4, 1951 (2013).

[4] A. Tredicucci and M. S. Vitiello, IEEE J. Sel. Top. Quantum Electron. 20, 130 (2014).

[5] V. Ryzhii, A. Satou, T. Otsuji, M. Ryzhii, V. Mitin, and M. S. Shur, J. Appl. Phys. 116, 114504 (2014).

[6] X. Cai, A. B. Sushkov, R. J. Suess, M. M. Jadidi, G. S. Jenkins, L. O. Nyakiti, R. L. Myers-Ward, S. Li, J. Yan, D. K. Gaskill et al., Nat. Nanotechnol. 9, 814 (2014).

[7] M. Mittendorff, J. Kamann, J. Eroms, D. Weiss, C. Drexler, S. D. Ganichev, J. Kerbusch, A. Erbe, R. J. Suess, T. E. Murphy et al., Opt. Express 23, 28728 (2015).

[8] A. Rogalski, M. Kopytko, and P. Martyniuk, Appl. Phys. Rev. 6, 021316 (2019).

[9] M. Glazov and S. Ganichev, Phys. Rep. 535, 101 (2014).

[10] J. You, S. Bongu, Q. Bao, and N. Panoiu, Nanophotonics 8, 63 (2018).

[11] E. L. Ivchenko and S. D. Ganichev, Pisma v ZhETF 93, 752 (2011) [JETP Lett. 93, 673 (2011)].

[12] S. Denisov, S. Flach, and P. Hänggi, Phys. Rep. 538, 77 (2014).

[13] D. Bercioux and P. Lucignano, Rep. Prog. Phys. 78, 106001 (2015).

[14] C. O. Reichhardt and C. Reichhardt, Annu. Rev. Condens. Matter Phys. 8, 51 (2017).

[15] Y. Y. Kiselev and L. E. Golub, Phys. Rev. B 84, 235440 (2011).

[16] L. Ermann and D. L. Shepelyansky, Eur. Phys. J. B 79, 357 (2011).

[17] S. V. Koniakhin, Eur. Phys. J. B 87, 216 (2014).

[18] C. Jiang, V. A. Shalygin, V. Y. Panevin, S. N. Danilov, M. M. Glazov, R. Yakimova, S. Lara-Avila, S. Kubatkin, and S. D. Ganichev, Phys. Rev. B 84, 125429 (2011).

[19] A. Tomadin, A. Tredicucci, V. Pellegrini, M. S. Vitiello, and M. Polini, Appl. Phys. Lett. 103, 211120 (2013).

[20] A. V. Muraviev, S. L. Rumyantsev, G. Liu, A. A. Balandin, W. Knap, and M. S. Shur, Appl. Phys. Lett. 103, 181114 (2013).

[21] D. Spirito, D. Coquillat, S. L. D. Bonis, A. Lombardo, M. Bruna, A. C. Ferrari, V. Pellegrini, A. Tredicucci, W. Knap, and M. S. Vitiello, Appl. Phys. Lett. 104, 061111 (2014).

[22] X. Cai, A. B. Sushkov, M. M. Jadidi, L. O. Nyakiti, R. L. Myers-Ward, D. K. Gaskill, T. E. Murphy, M. S. Fuhrer, and H. D. Drew, Nano Lett. 15, 4295 (2015).

[23] L. Wang, X. Chen, and W. Lu, Nanotechnology 27, 035205 (2015).

[24] G. Auton, D. B. But, J. Zhang, E. Hill, D. Coquillat, C. Consejo, P. Nouvel, W. Knap, L. Varani, F. Teppe et al., Nano Lett. 17, 7015 (2017).

[25] D. A. Bandurin, D. Svintsov, I. Gayduchenko, S. G. Xu, A. Principi, M. Moskotin, I. Tretyakov, D. Yagodkin, S. Zhukov, T. Taniguchi et al., Nat. Commun. 9, 5392 (2018).

[26] S. D. Ganichev, D. Weiss, and J. Eroms, Ann. Phys. 529, 1600406 (2018).

[27] T. Otsuji, T. Watanabe, S. A. B. Tombet, A. Satou, W. M. Knap, V. V. Popov, M. Ryzhii, and V. Ryzhii, IEEE Trans. Terahertz Sci. Technol. 3, 63 (2013).
[28] A. V. Nalitov, L. E. Golub, and E. L. Ivchenko, Phys. Rev. B 86, 115301 (2012).

[29] P. Olbrich, J. Kamann, M. König, J. Munzert, L. Tutsch, J. Eroms, D. Weiss, M.-H. Liu, L. E. Golub, E. L. Ivchenko et al., Phys. Rev. B 93, 075422 (2016).

[30] V. V. Popov, Appl. Phys. Lett. 108, 261104 (2016).

[31] D. Fateev, K. Mashinsky, J. Sun, and V. Popov, Solid-State Electron. 157, 20 (2019).

[32] I. V. Rozhansky, V. Y. Kachorovskii, and M. S. Shur, Phys. Rev. Lett. 114, 246601 (2015).

[33] C. Drexler, S. A. Tarasenko, P. Olbrich, J. Karch, M. Hirmer, F. Müller, M. Gmitra, J. Fabian, R. Yakimova, S. Lara-Avila et al., Nat. Nanotech. 8, 104 (2013).

[34] E. L. Ivchenko, Y. B. Lyanda-Geller, and G. E. Pikus, Ferroelectrics 83, 19 (1988).

[35] V. I. Falko, Fiz. Tverd. Tela 31, 29 (1989) [Sov. Phys. Solid State 31, 561 (1989)].

[36] V. V. Bel'kov, S. D. Ganichev, E. L. Ivchenko, S. A. Tarasenko, W. Weber, S. Giglberger, M. Olteanu, H. P. Tranitz, S. N. Danilov, P. Schneider et al., J. Phys.: Condens. Matter 17, 3405 (2005).

[37] S. A. Tarasenko, Phys. Rev. B 83, 035313 (2011).

[38] L. Wang, I. Meric, P. Y. Huang, Q. Gao, Y. Gao, H. Tran, T. Taniguchi, K. Watanabe, L. M. Campos, D. A. Muller et al., Science 342, 614 (2013).

[39] Z.-D. Kvon, S. N. Danilov, N. N. Mikhailov, S. A. Dvoretsky, W. Prettl, and S. D. Ganichev, Physica E 40, 1885 (2008).

[40] S. D. Ganichev, S. A. Tarasenko, V. V. Bel'kov, P. Olbrich, W. Eder, D. R. Yakovlev, V. Kolkovsky, W. Zaleszczyk, G. Karczewski, T. Wojtowicz, and D. Weiss, Phys. Rev. Lett. 102, 156602 (2009).

[41] P. Olbrich, C. Zoth, P. Vierling, K.-M. Dantscher, G. V. Budkin, S. A. Tarasenko, V. V. Bel'kov, D. A. Kozlov, Z. D. Kvon, N. N. Mikhailov et al., Phys. Rev. B 87, 235439 (2013).

[42] S. Ganichev, Physica B 273-274, 737 (1999).

[43] E. Ziemann, S. D. Ganichev, W. Prettl, I. N. Yassievich, and V. I. Perel, J. Appl. Phys. 87, 3843 (2000).

[44] W. Weber, L. E. Golub, S. N. Danilov, J. Karch, C. Reitmaier, B. Wittmann, V. V. Bel'kov, E. L. Ivchenko, Z. D. Kvon, N. Q. Vinh et al., Phys. Rev. B 77, 245304 (2008).

[45] Due to the fact that the first (TG1) and second (TG2) top gate electrodes are at the same distance from the graphene layer, we expect that both gates equally change the carrier concentration in graphene. Consequently, the oscillations period for the curves obtained by varying $U_{\mathrm{TG} 1}$ and $U_{\mathrm{TG} 2}$ should be the same. Comparing extrema positions for the gate voltages range with wellresolved oscillations in the both curves support this conclusion. This allows one the comparison of the relative oscillations sign. We note that the detailed analysis of this plot is limited to a rather small range of the gate voltages, because for top gate values close to zero $\left(\left|U_{\mathrm{TG} 1, \mathrm{TG} 2}\right|<1 \mathrm{~V}\right)$ the ratchet signal is dominated by the build-in asymmetry and for $U_{\mathrm{TG} 2}<-2 \mathrm{~V}$ one the traces becomes noisy.

[46] G. V. Budkin, L. E. Golub, E. L. Ivchenko, and S. D. Ganichev, JETP Lett. 104, 649 (2016). 
[47] P. Faltermeier, G. V. Budkin, J. Unverzagt, S. Hubmann, A. Pfaller, V. V. Bel'kov, L. E. Golub, E. L. Ivchenko, Z. Adamus, G. Karczewski et al., Phys. Rev. B 95, 155442 (2017).

[48] P. Faltermeier, G. Budkin, S. Hubmann, V. Bel'kov, L. Golub, E. Ivchenko, Z. Adamus, G. Karczewski, T. Wojtowicz, D. Kozlov et al., Physica E 101, 178 (2018).
[49] U. Briskot, I. A. Dmitriev, and A. D. Mirlin, Phys. Rev. B 87, 195432 (2013).

[50] C. Zoth, P. Olbrich, P. Vierling, K.-M. Dantscher, V. V. Bel'kov, M. A. Semina, M. M. Glazov, L. E. Golub, D. A. Kozlov, Z. D. Kvon et al., Phys. Rev. B 90, 205415 (2014).

[51] D. V. Fateev, K. V. Mashinsky, and V. V. Popov, Appl. Phys. Lett. 110, 061106 (2017). 